\section{OPEN ACCESS}

Edited by:

Xiaogang Wu,

University of Texas MD Anderson

Cancer Center, United States

Reviewed by:

Bhanwar Lal Puniya,

University of Nebraska-Lincoln,

United States

Georges Nemer,

American University of Beirut,

Lebanon

${ }^{*}$ Correspondence:

Lokesh P. Tripathi

lokesh@nibiohn.go.jp

Mari N. Itoh

mari@nibiohn.go.jp

Kenji Mizuguchi

kenji@nibiohn.go.jp

Specialty section:

This article was submitted to

Systems Biology,

a section of the journal

Frontiers in Genetics

Received: 22 July 2020 Accepted: 28 October 2020 Published: 10 December 2020

Citation:

Tripathi LP, Itoh MN, Takeda Y, Tsujino K, Kondo Y, Kumanogoh A and Mizuguchi K (2020) Integrative Analysis Reveals Common and Unique Roles of Tetraspanins

in Fibrosis and Emphysema.

Front. Genet. 11:585998. doi: $10.3389 /$ fgene. 2020.585998

\title{
Integrative Analysis Reveals Common and Unique Roles of Tetraspanins in Fibrosis and Emphysema
}

\author{
Lokesh P. Tripathi1,2*, Mari N. Itoh ${ }^{1,2 *}$, Yoshito Takeda ${ }^{3}, K_{\text {Kazuyuki Tsujino }}^{3}$, \\ Yasushi Kondo ${ }^{4}$, Atsushi Kumanogoh ${ }^{3}$ and Kenji Mizuguchi ${ }^{1,2,5 *}$
}

${ }^{1}$ National Institutes of Biomedical Innovation, Health and Nutrition, Ibaraki, Japan, ${ }^{2}$ Artificial Intelligence Center for Health and Biomedical Research (ArCHER), National Institutes of Biomedical Innovation, Health and Nutrition, Ibaraki, Japan, ${ }^{3}$ Department of Respiratory Medicine, Allergy and Rheumatic Diseases, Osaka University Graduate School of Medicine, Suita, Japan, ${ }^{4}$ Research Division, Sumitomo Dainippon Pharma Co., Ltd., Osaka, Japan, ${ }^{5}$ Institute for Protein Research, Osaka University, Suita, Japan

While both chronic obstructive pulmonary disease (COPD) and idiopathic pulmonary fibrosis (IPF) are multifactorial disorders characterized by distinct clinical and pathological features, their commonalities and differences have not been fully elucidated. We sought to investigate the preventive roles of tetraspanins Cd151 and Cd9 -that are involved in diverse cellular processes in lung pathophysiology- in pulmonary fibrosis and emphysema, respectively, and to obtain a deeper understanding of their underlying molecular mechanisms toward facilitating improved therapeutic outcomes. Using an integrative approach, we examined the transcriptomic changes in the lungs of Cd151and Cd9-deficient mice using functional-enrichment-analysis, pathway-perturbationanalysis and protein-protein-interaction (PPI) network analysis. Circadian-rhythm, extracellular-matrix (ECM), cell-adhesion and inflammatory responses and associated factors were prominently influenced by Cd151-deletion. Conversely, cellular-junctions, focal-adhesion, vascular-remodeling, and TNF-signaling were deeply impacted by Cd9deletion. We also highlighted a "common core" of factors and signaling cascades that underlie the functions of both Cd151 and Cd9 in lung pathology. Circadian dysregulation following Cd151-deletion seemingly facilitated progressive fibrotic lung phenotype. Conversely, TGF- $\beta$ signaling attenuation and TNF-signaling activation emerged as potentially novel functionaries of Cd9-deletion-induced emphysema. Our findings offer promising avenues for developing novel therapeutic treatments for pulmonary fibrosis and emphysema.

Keywords: tetraspanins, COPD, IPF, integrative gene expression analysis, cellular networks, signaling pathways, systems biology, biomarker discovery

\section{INTRODUCTION}

COPD is a progressive lung disease that is characterized by the exposure to a noxious agent (such as cigarette smoke) resulting in airflow limitation that is not fully reversible. A subset of COPD patients sustain the destruction of lung elastin and other ECM proteins, apoptosis of alveolar cells, or repair failure that leads to airspace enlargement, a characteristic of emphysema (Vogelmeier et al., 2017). IPF, on the other hand, is characterized by interstitial pneumonia, uncontrolled and progressive ECM-deposition, and 
abnormal alveolar remodeling (Raghu et al., 2011). Combined pulmonary fibrosis and emphysema (CPFE) is recognized as a clinical syndrome that is defined by the co-existence of emphysema and parenchymal fibrosis in the patient (Cottin et al., 2005). While CPFE patients have distinct clinical features and possibly different outcomes, their pathogenesis remains unclear.

Tetraspanins are integral membrane proteins with four transmembrane helices that participate in varied cellular and physiological processes (Charrin et al., 2014; Termini and Gillette, 2017). Some tetraspanins such as Cd151 are widely expressed in a variety of cancer types and positively regulate tumor progression (Kumari et al., 2015). Cd9, in contrast, negatively regulates tumor progression.

We had previously demonstrated that Cd151 is necessary for the integrity of the alveolar epithelial cells (AECs); Cd151 deletion had resulted in the activation of phosphorylated Smad2 and EMT-like changes that may potentially contribute to the development of pulmonary fibrosis in the mouse lung. Indeed, it was further observed that Cd151-knockout mice (Cd151KO) had spontaneously developed age-related pulmonary fibrosis (Tsujino et al., 2012). Conversely, Cd9-deletion exacerbated lipopolysaccharide (LPS) induced lung inflammation that was characterized by macrophage infiltration and the overproduction of TNF- $\alpha$ and matrix metalloproteinsases (MMPs). Moreover, $\mathrm{Cd} 9$-deficient mice $(\mathrm{Cd} 9 \mathrm{KO})$ were prone to progressive emphysema, a major pathological component of COPD (Takeda et al., 2008; Suzuki et al., 2009). We further demonstrated that Cd9/Cd81 double-knockout mice spontaneously developed pulmonary emphysema (Jin et al., 2018) and that Cd9 likely performs protective roles in lung inflammation and emphysema.

Here, we have employed an integrative bioinformatics approach to investigate the transcriptomic changes in the mouse lung tissue underlying the deletions of $\mathrm{Cd} 151$ and $\mathrm{Cd} 9$ to (a) gain a perspective of a corresponding phenomenon in context of the human lung and; (b) to better understand the preventive roles of $\mathrm{Cd} 151$ and $\mathrm{Cd} 9$ in pulmonary fibrosis and emphysema, respectively (Figure 1A). First, we examined the significantly differentially expressed genes (sDEGs) in Cd151KO and Cd9KO lungs for enriched biological themes to highlight key cellular processes that were dysregulated by Cd151- and Cd9deletions. Next, we investigated the perturbative impacts of these dysregulated gene expressions on cellular signaling pathways. We finally investigated these transcriptomic changes in the context of human orthologous PPI networks (PPINs) to highlight affected signaling cascades and probable crosslinks between them.

Our analysis has led to a deeper understanding of the molecular mechanisms underlying lung fibrosis and emphysema and provided promising avenues for improved therapeutic applications. We describe our analysis and discuss our observations below.

\section{MATERIALS AND METHODS}

\section{Animal Models}

Cd151KO and Cd9KO were generated as before (Tsujino et al., 2012). All animal experiments were approved by the Animal
Care and Use Committee of Osaka University, and all of the animal procedures were performed in accordance with the Osaka University guidelines on animal care.

\section{Sample Preparation and Microarray Gene Expression Analysis}

Lungs removed from mice (WT $(n=4)$, CD151 KO $(n=5)$, and $\mathrm{CD} 9 \mathrm{KO}(n=5)$, at 20 weeks of age) were immersed in RNA-Later solution (Invitrogen) and stored at $-80^{\circ} \mathrm{C}$ until RNA extraction. The lungs were transferred into QIAzol lysis reagent (Qiagen) and disrupted and homogenized using a TissueLyser homogenizer (Qiagen). Total RNA was extracted from the lysates using the RNeasy mini kits (Qiagen) according to the manufacturer's instructions.

Gene expression profiles were obtained from 100 ng total RNA per sample using GeneChip Mouse Genome 430 2.0 Arrays and GeneChip 3'-IVT Express Kit (Affymetrix). The signal intensities derived from probe sets were normalized among samples using the Microarray Suite (MAS) 5.0 algorithm implemented in the Expression Console software (Affymetrix), where signal intensities of all probe sets were linearly scaled as their trimmed mean values to be 500. QC metrics reported by the software were confirmed to be within normal ranges.

The differences between the two groups were evaluated using two-sided Student's $t$-test and $p \leq 0.05$ were considered statistically significant. Probesets were judged to be differentially expressed if the Fold change (FC) $\geq 1.5$ (upregulated) or FC $\leq 0.6667$ (downregulated), in $\mathrm{Cd} 151 \mathrm{KO} / \mathrm{Cd} 9 \mathrm{KO}$ vs. WT. These probesets were transformed into sDEG-sets for subsequent analyses; for genes with multiple probesets, the highest FC values were selected.

\section{Gene Set Functional Enrichment (GSFE) Analysis}

Cd151KO and Cd9KO sDEGs were examined with TargetMine data analysis platform ${ }^{1}$ (Chen et al., 2011, 2016). The enrichment of specific KEGG pathways (Kanehisa et al., 2016), Reactome pathways (Fabregat et al., 2016) and Gene Ontology (GO) associations was estimated using Fisher's exact test (Gene Ontology Consortium, 2015). Inferred $p$-values were further adjusted for multiple-test-correction to control the falsediscovery-rate using the Benjamini-Hochberg procedure (Benjamini et al., 2001), and the annotations/pathways were considered significant if the adjusted $p$-values $\leq 0.05$. The enriched associations were visualized as heatmaps with the TargetMine auxiliary toolkit (Chen et al., 2016).

\section{Co-expression Gene Module Network Analysis}

To identify co-expressed genes specifically associated with $\mathrm{Cd} 151 \mathrm{KO}$ or $\mathrm{Cd} 9 \mathrm{KO}$, we employed the weighted gene coexpression network analysis (WGCNA) package in R (Langfelder and Horvath, 2008). A unified set of $\mathrm{Cd} 151 \mathrm{KO}$ and $\mathrm{Cd} 9 \mathrm{KO}$

\footnotetext{
${ }^{1}$ http://targetmine.mizuguchilab.org
} 
A

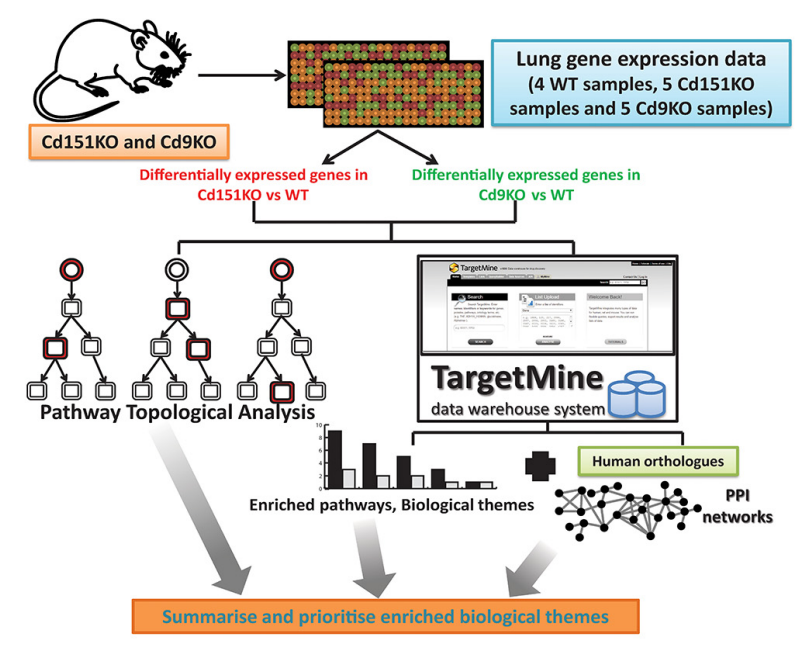

B

Cd151KO-1.5FC

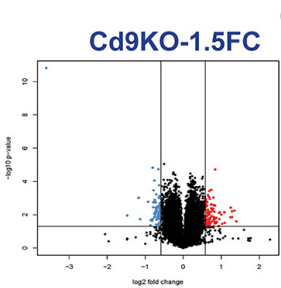

C Co9ko

Cd151KO

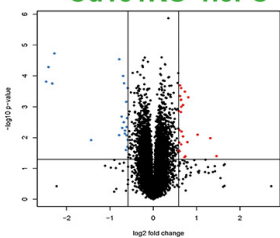

43 Genes $(28$

Up/15 down)

D

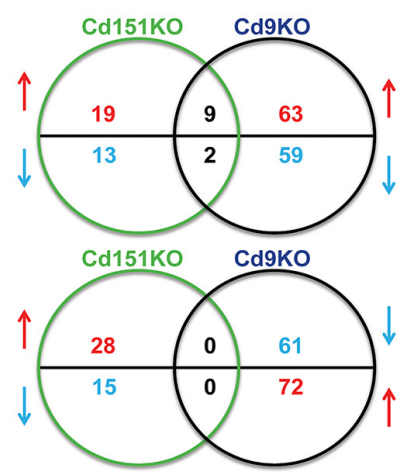

133 Genes (72

up/61 down)

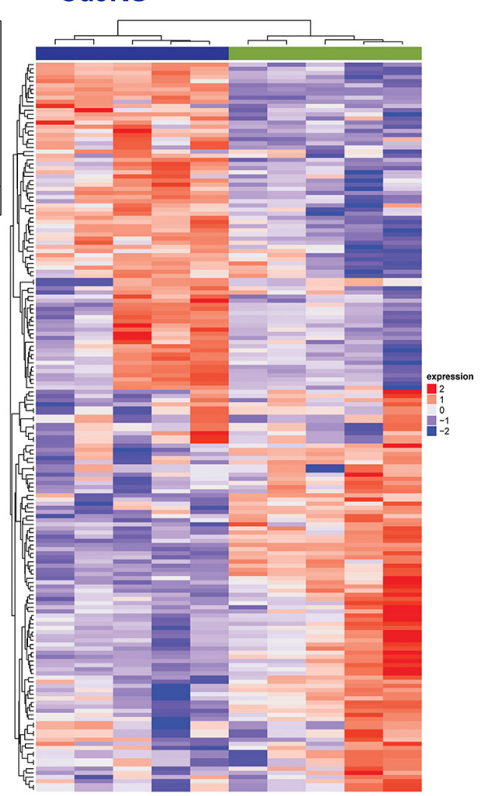

E

\begin{tabular}{|c|c|c|c|c|c|}
\hline Probe ID & Gene ID & Gene Symbol & Name & $\begin{array}{l}\text { Fold change } \\
\text { Cd1511\%o }\end{array}$ & $\begin{array}{l}\text { Fold change } \\
\text { Cdgro }\end{array}$ \\
\hline \multicolumn{6}{|c|}{ Upregulated genes } \\
\hline 1424737_at & 21835 & Thrsp & thyroid hormone responsive & 1.927859456 & 1.770595421 \\
\hline 1416121_at & 16948 & Lox & lysyl oxidase & 1.670810994 & 1.788609495 \\
\hline 1453351_at & 57246 & $T \mathrm{~b} \times 20$ & T-box 20 & 1.649670004 & 1.74187999 \\
\hline 1427056_at & 235130 & Adamts 15 & $\begin{array}{l}\text { a disintegrin-like and metallopeptidase (reprolysin type) with } \\
\text { thrombospondin type } 1 \text { motif, } 15\end{array}$ & 1.592328518 & 1.930829713 \\
\hline 1422651_at & 11450 & Adipoq & adiponectin, $\mathrm{C} 1 \mathrm{Q}$ and collagen domain containing & 1.561764894 & 1.529442072 \\
\hline 1436453_at & 101918 & BB144871 & expressed sequence BB144871 & 1.526558048 & 1.903804544 \\
\hline 1420855_at & 13717 & Eln & elastin & 1.522211876 & 1.626248061 \\
\hline 1428223_at & 76574 & Misdza & major facilitator superfamily domain containing $2 \mathrm{~A}$ & 1.507399865 & 1.792199829 \\
\hline 1417803_at & 66183 & Sptssb & serine palmitoyltransferase, small subunit B & 1.50366407 & 1.541466319 \\
\hline \multicolumn{6}{|c|}{ Downregulated genes } \\
\hline 1415969_s_at & 16483 & Кар & kidney androgen regulated protein & 0.370243283 & 0.360568152 \\
\hline 1438033_at & 21685 & Tef & thyrotroph embryonic factor & 0.617127023 & 0.66425 \\
\hline
\end{tabular}

FIGURE 1 | (A) A multi-omics framework to investigate the transcriptomic changes underlying the deletion of Cd151 and Cd9. (B) Volcano plots for Cd151KO and Cd9KO sDEGs (1.5FC). Upregulated probes (genes) are highlighted in red, whereas the downregulated probes (genes) are highlighted in blue. (C) A heatmap of the significantly fluctuated gene expression levels in Cd151KO (green) and Cd9KO (deep blue). Red indicates relative higher expression, blue indicates relative lower expression; values in each row are normalized gene expression. Both rows and columns were hierarchically clustered and the results were illustrated as dendrograms. (D) Cd151KO (green) and Cd9KO (deep blue) sDEGs show very little overlap in either upregulated genes (red upward arrow) or downregulated genes (blue downward arrow). (E) The similarly affected genes in Cd151KO and Cd9KO indicating convergent underlying mechanisms. 
sDEGs was used for the estimation of Cd151KO or Cd9KOspecific gene modules. The blockwise-Module function was used with the default parameters and power $=7$; deepSplit $=2$. The selected genes were further examined for functional enrichment analysis.

\section{Pathway-Perturbation Analysis}

We employed Signaling Pathway Impact Analysis (SPIA) package in $\mathrm{R}$ (Tarca et al., 2009) to analyse KEGG signalling pathways in mouse. SPIA combines data from gene expression measurements with the classical pathway enrichment (overrepresentation) analysis and the actual perturbation of a given pathway under specific conditions. SPIA achieves this by estimating a global pathway significance $p$-value ( $\mathrm{pG}$ ) that combines pathway enrichment ( $\mathrm{pNDE}$ ) and perturbation $p$-values (pPERT), thereby providing a robust assessment of the impact of differential gene expression in the biological processes under study.

\section{Construction of Orthologous sDEGs PPINs}

PPI networks for Cd151KO and Cd9KO sDEGs were constructed using TargetMine (see Supplementary Material for details). Network components were visualized with Cytoscape (Cline et al., 2007) and network hubs and bottlenecks (Chen et al., 2016) were assigned using TargetMine.

\section{RESULTS}

\section{Cd151KO and Cd9KO Show Distinct Transcriptomic Profiles}

Forty three sDEGs were upregulated (28 genes) or downregulated (15 genes) in Cd151KO. Likewise, 133 (72 upregulated and 61 downregulated) sDEGs were identified in Cd9KO (Figure 1B and Supplementary Tables S1A,B). Cd151KO and Cd9KO displayed dissimilar transcriptomic profiles (Figure 1C). Only nine genes (Adamts15, Adipoq, BB144871, Eln, Lox, Mfsd2a, Sptssb, Tbx20, and Thrsp) were upregulated and two genes (Kap, Tef) were downregulated in both Cd151 KO and Cd9KO (Figures 1D,E and Supplementary Table S1).

\section{GSFE Analysis of Cd151KO and Cd9KO sDEGs}

First, we investigated the Cd151KO and Cd9KO sDEGs for enriched biological themes.

\section{Cd151KO sDEGs Were Enriched in Circadian Rhythm, ECM, and Collagen Deposition Pathways GSFE (Figure 2A and Supplementary Table S2) with Cd151KO sDEGs revealed that 12 sDEGs (seven upregulated and five downregulated; Figure 2B) were mapped to pathways associated with circadian rhythm, regulation of ECM and collagen deposition (Figures 2A,B, Supplementary Table S2, and Supplementary Material). \\ Specifically, circadian clock-associated genes Cry1 (0.58FC), Per2 (0.65FC), and Per3 (0.66FC) were downregulated in}

Cd151KO, but not in Cd9KO (Cry1 was marginally upregulated 1.17 -fold in $\mathrm{Cd} 9 \mathrm{KO})$. Conversely, circadian regulator Arntl (Bmal1) was elevated 2.4-fold in Cd151KO (1.01FC in Cd9KO) (Supplementary Table S1A). Additionally, Lox (lysysl oxidase), an ECM modifying enzyme, Col3a1 (collagen, type III, alpha I), Mfap5 (microfibrillar associated protein 5) and $E \ln$ (elastin), an ECM protein and a key component of lung tissues, were upregulated 1. 67-, 1. 56-, 1. 53-, and 1.52-fold, respectively, in Cd151KO (Supplementary Table S1 and Figure 2B).

The WGCNA algorithm (Langfelder and Horvath, 2008) (Supplementary Material) analysis also identified 31 Cd151KO-specific sDEGs that included key "Circadian rhythm" components Arntl, Cry1, Npas2, Per2, and Per3.

\section{Cd9KO sDEGs Were Significantly Associated With ECM}

Biological enrichment analysis (Figure 2C and Supplementary Table S2) of the Cd9KO sDEGs revealed that 52 sDEGs (27 upregulated and 25 downregulated; Figure 2D) were mapped to five GO (Slim) Biological Process terms ( $p \leq 0.05)$ : "homeostasis," "circulatory system process," "extracellular matrix organization," "cell differentiation" and "anatomical structure development" (Supplementary Table S2).

Cd9-deletion results in aberrations in cell matrix (Suzuki et al., 2009). Lox, Mkx, Eln, and Mmp13, a matrix metalloproteinsase, were upregulated 1.78-, 1.67-, 1.62-, and 1.55-fold, respectively; conversely, Eng (Endoglin), a glycoprotein, and Ndnf, a neutrotrophic factor, were downregulated 0.66- and 0.55-fold, respectively, in Cd9KO (Supplementary Table S1B).

Cd9-deletion is also associated with diminished angiogenetic effects (Iwasaki et al., 2013). Accordingly, many factors dysregulated in Cd9KO were known to be dysregulated in airway obstruction, emphysema and COPD (Miller et al., 2009; Llinas et al., 2011; Brightling, 2013). Among these factors, Adipoq (Adiponectin), FggFgg (fibrinogen gamma chain), Gclc (glutamate-cysteine ligase, catalytic subunit), and Calca (Calcitonin) were upregulated 1. 52-, 1. 64-, 1. 63-, and 1.5-fold, respectively, in Cd9KO (Supplementary Table S1B and Figure 2D). Conversely, Glp1r (glucagon-like peptide 1 receptor) and Ednrb (endothelin receptor type B) were downregulated 0.66 - and 0.65 -fold, respectively, in $\mathrm{Cd} 9 \mathrm{KO}$ (Supplementary Table S1B).

\section{Signaling Pathway Perturbations in Cd151KO and Cd9KO}

Next, we investigated the perturbative effects of Cd151- and Cd9deletions on signaling pathways using SPIA (Supplementary Material, Figures 3, 4, and Supplementary Figures S1, S3).

\section{Cd151-Deletion Activated Circadian Rhythm, ECM, and Focal Adhesion Pathways}

SPIA revealed that "Circadian rhythm" was "Activated" in Cd151KO $\left(\right.$ pGFWER $\left.=1.55 \times 10^{-9}\right)($ Supplementary Figure S3 and Supplementary Table S3). Circadian rhythm is an internal biological clock that modulates the daily physiology and behavior of organisms. This 24-h coordination is achieved by intracellular transcription feedback loops; E-box-driven period (PER) and 
A

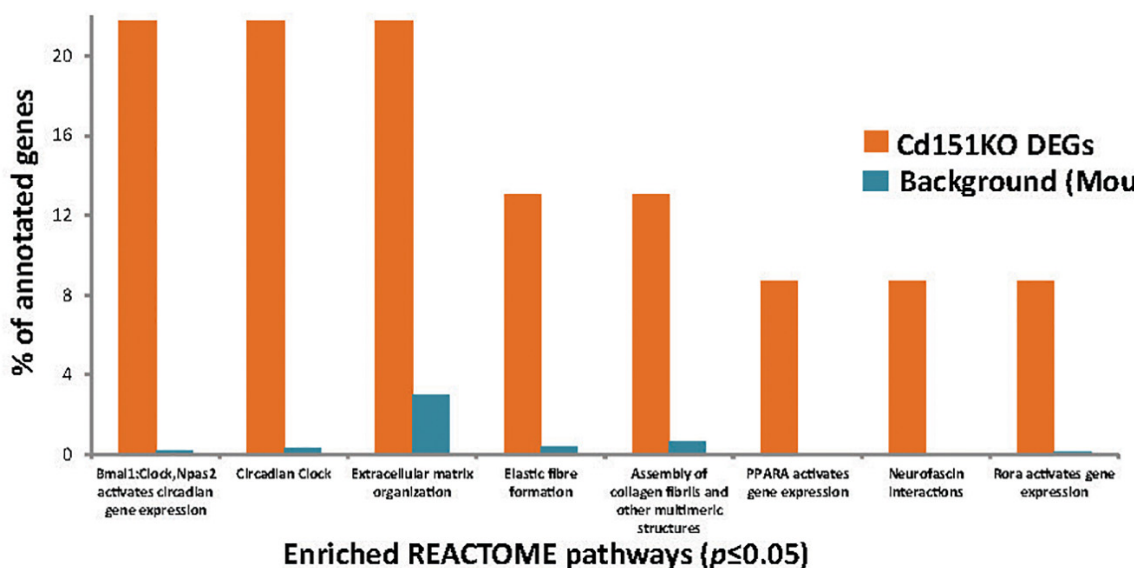

Enriched REACTOME pathways $(p \leq 0.05)$

B
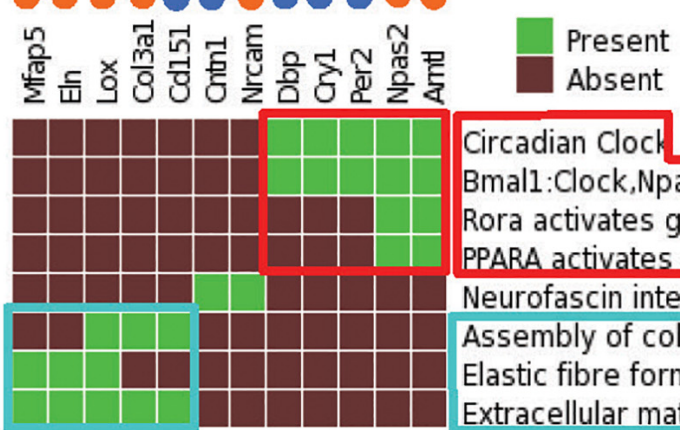

Circadian Clock

Circadian rhythm associated pathways

Bmal1:Clock,Npas2 activates circadian gene expression

Rora activates gene expression

PPARA activates gene expression

Neurofascin interactions

Assembly of collagen fibrils and other multimeric structures

Elastic fibre formation Modulation of extracellular matrix and

Extracellular matrix organization collagen deposition

C

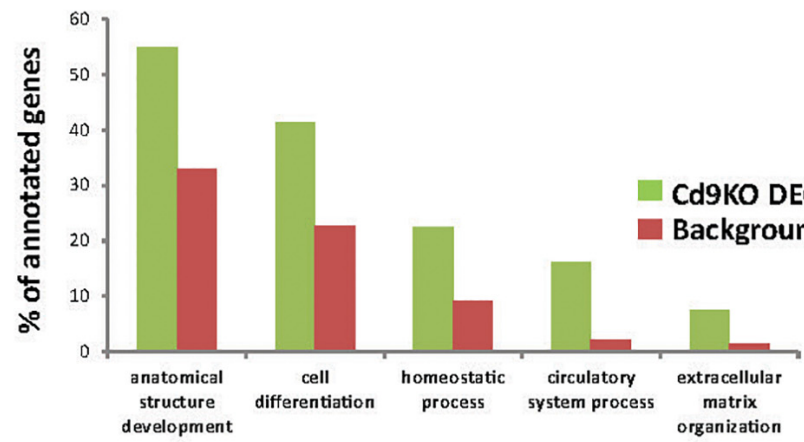

Enriched Gene Ontology Slim (Biological Process) associations $(p \leq 0.05)$

D

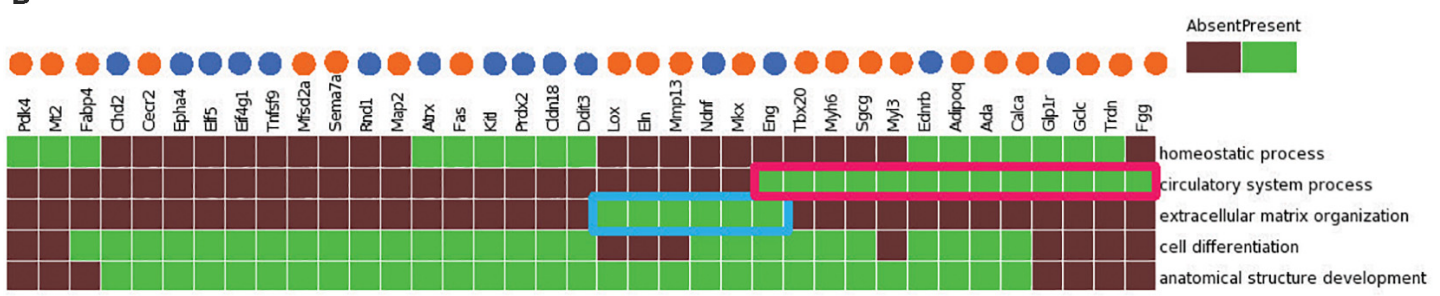

FIGURE 2 | (A) Enriched Reactome pathways ( $0 \leq 0.05$ ) identified in Cd151KO 1.5FC sDEGs. For clarity, only select Reactome pathways are displayed. (B) Cd151KO sDEGs were distinctly enriched in Reactome pathways associated with circadian rhythm (red box) and extracellular matrix (azure box). (C) Enriched GO Slim biological process (BP) terms $(p \leq 0.05)$ identified in Cd9KO 1.5FC sDEGs. (D) Cd9KO sDEGs were significantly associated with enriched GO Slim BP terms "circulatory system process" (red box) and "extracellular matrix" (blue box); for clarity, only a subset of the heatmap is highlighted here. In (B,D), the upregulated genes are indicated by orange spheres and downregulated genes are indicated by blue spheres. 
A

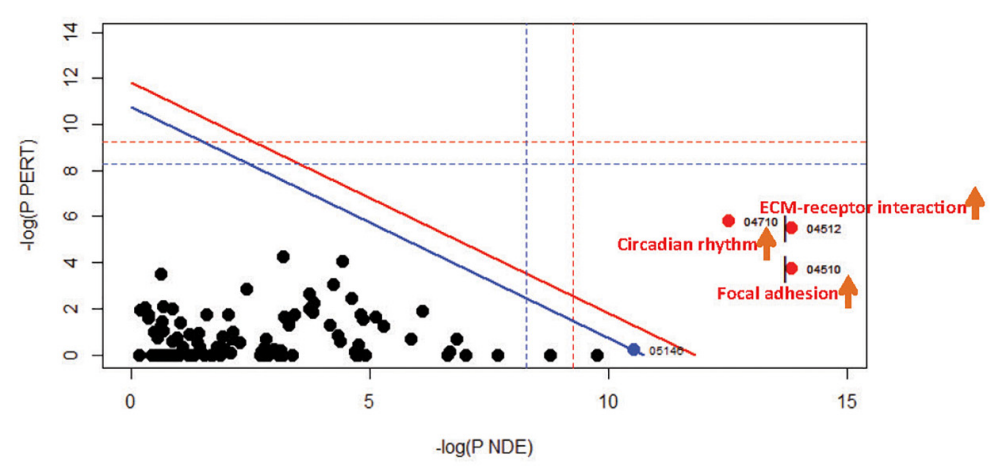

B

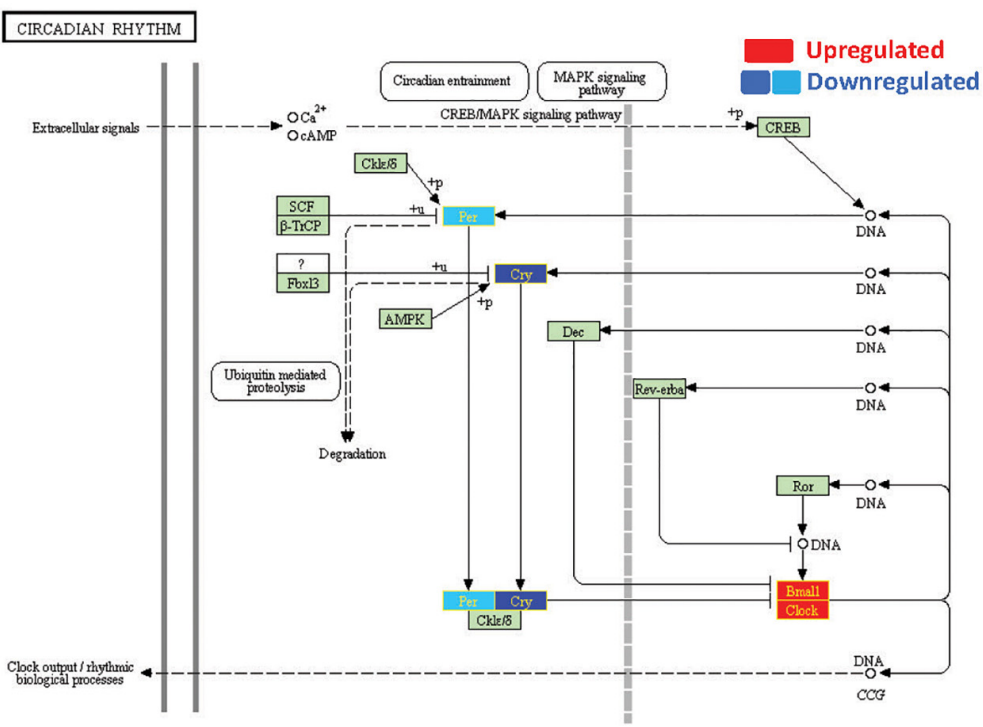

C

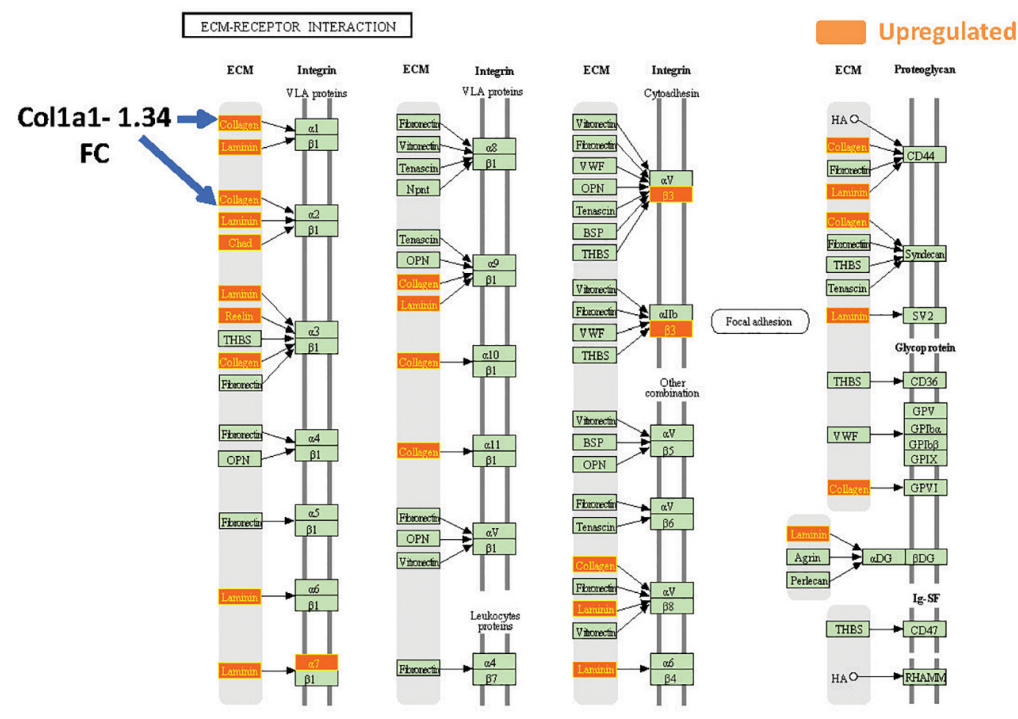

FIGURE 3 | SPIA identified pathway perturbations in Cd151KO. (A) SPIA two-way evidence plots for Cd151 1.2FC sDEGs. Significantly perturbed pathways are highlighted in red. "Activated" pathways are tagged with a red upward arrow. (B) KEGG pathway hsa04710: "Circadian rhythm" was markedly activated in Cd151KO 1.2FC sDEGs. Upregulated genes are highlighted in red and the downregulated genes are highlighted in dark blue (Cry) and light blue (Per), rectangles, respectively, with gene labels within highlighted in yellow. (C) KEGG pathway hsa04512: "ECM-receptor interaction" was activated in Cd151KO Upregulated genes are highlighted in Orange rectangles; their corresponding gene labels within the rectangles are highlighted in yellow. 
cryptochrome (CRY) proteins heterodimerize and impede the transcriptional activator complex CLOCK/BMAL1 to repress their own transcription (Albrecht, 2012; Peek et al., 2015). SPIA suggested an activation of "Circadian rhythm" pathway (and consequently increased clock output) due to downregulation of Cry 1 and Per 2 and upregulation of the transcriptional activators Arntl1 and Npas2 (Garavoglia et al., 1993; Haque et al., 2010; Landgraf et al., 2016) following Cd151-deletion (Figure 3B).

SPIA also highlighted that "ECM-receptor interaction" and "Focal adhesion" pathways were "Activated" (Figure 3A and Supplementary Table S3) in Cd151KO (with 1.2FC sDEGs; Supplementary Material). Therefore, Cd151-deletion resulted in increased collagen- and ECM-deposition via upregulation of Col3a1 (1.56FC) and Lamc2 (laminin, gamma; 1.23FC). The increased ECM-deposition induced a loosening of cellular adhesions chiefly via upregulation of cell surface proteins including Intga7 (integrin alpha 7; 1.25FC) and Intgb3 (integrin beta $3 ; 1.26 \mathrm{FC}$ ). The activation of "ECM-receptor interaction" and "Focal adhesion" pathways manifested in the upregulation of cell proliferation factor Cycd (cyclin D; $1.23 \mathrm{FC}$ ) and in downregulation of apoptosis-inhibitor Xiap (X-linked inhibitor of apoptosis; 0.8FC) (Figure $3 \mathrm{C}$ and Supplementary Tables S1A, S3).

Cd9-Deletion Perturbed ECM, Cellular Junction, and Inflammatory Immune Response Pathways

SPIA with Cd9KO 1.2FC sDEGs (Supplementary Figure S1) highlighted ten significantly impacted pathways (five "Activated" and five "Inhibited"; Supplementary Material) in Cd9KO (Figure 4A and Supplementary Table S3).

Cd9-deletion significantly impacted pro-inflammatory signaling, chiefly via the activations of "MAPK signaling pathway" (mmu04010; pGFWER = 0.000834) and "Chemokine signaling pathway" (mmu04062; pGFWER = 0.00315), which are consistent with increased lung inflammation and pro-inflammatory cytokines in Cd9KO (Suzuki et al., 2009).

Cd9-deletion also significantly "Inhibited" "Tight Junction" pathway, chiefly because Cldn18 (Claudin18), an integral membrane protein, was decreased 0.65-fold (Supplementary Table S1B); Cldn18 was implicated as a key factor in lung barrier and alveolar junction functions and in lung inflammation (Schlingmann et al., 2015; Shimobaba et al., 2016). An examination of the "Tight Junction" pathway map (Figure 4B) suggested that a decrease in Cldn18 function may likely impede tight junction formation, thereby affecting multiple downstream processes including cellular migration and motility (Figure 4B).

"TGF-beta signaling pathway" (mmu04350; pGFWER $=0.009$ ) was shown to be "Inhibited" in Cd9KO (Supplementary Table S3). Furthermore, Decorin, a Tgfb antagonist that sequesters Tgfb in the ECM thereby preventing it from interacting with its cell surface receptors (Jarvinen and Prince, 2015), was upregulated 1.35-fold in Cd9KO.

\section{Network Analysis of Cd151KO and Cd9KO sDEGs}

To circumvent the limited repertoire of mouse HCDPs (see Supplementary Methods), we retrieved the human orthologs of $\mathrm{Cd} 151 \mathrm{KO}$ and $\mathrm{Cd} 9 \mathrm{KO}$ sDEGs using TargetMine and inferred the Cd151KO and Cd9KO orthologous sDEGs networks (hereafter Cd151KO-network and Cd9KO-network, respectively) to examine the transcriptomic changes in a network context (Supplementary Figures S4A,B and Supplementary Material). In total, 38 human orthologs of 43 Cd151KO sDEGs $(88.3 \%)$ and 116 human orthologs of 133 Cd9KO sDEGs (87.2\%) were retrieved in this manner (Supplementary Table S4). The Cd151KO- and Cd9KO-networks were comprised of 110 genes, with 168 interactions between them, and of 347 genes, with 683 interactions between them, respectively (Supplementary Figure S4B and Supplementary Tables S5-S7).

\section{Cross-Talks Across Circadian Clock and Cell Cycle, Aeging, and Inflammation-Related Modules in Cd151KO-Network}

Cd151KO-network analysis revealed key interactions of circadian clock factors that were likely to be impacted by Cd151-deletion; PER2, the human ortholog of Per2 (downregulated in Cd151KO) interacted with CRY2 (Figure 5A), the human ortholog of Cry2 (unchanged in Cd151KO).

Network representations also suggested extensive cross-talks between Cell cycle, PI3-Akt signaling and aeging-related FoxO signaling pathways that were likely to be impacted by Cd151deletion. Ccnd1 (CyclinD1), a cell cycle regulator was upregulated 1.60-fold in Cd151KO (Supplementary Table S1). Ccnd1 human ortholog, CCND1 and its interactors CDK2, CDKN1A and CDKN1B in the Cd151KO-network were mapped to KEGG pathways "Cell cycle," "PI3K-Akt signaling pathway" and "FoxO signaling pathway"; two interactors (CDK4, CDK6) were mapped only to "Cell cycle" and "PI3K-Akt signaling pathway"; four (CDKN2A, PCNA, ORC4 and RB1) were mapped only to "Cell cycle"; BRCA1 was mapped to "PI3K-Akt signaling pathway" and STAT3 was mapped to "FoxO signaling pathway." Thus, CCND1, which was classified as a bottleneck-hub (Supplementary Table S7) appeared to mediate cross-talks across multiple pathways via multiple PPIs (Figure 5B).

Thrsp (thyroid hormone responsive), a lipid metabolism regulatory factor, was upregulated 1.92 -fold in $\mathrm{Cd} 151 \mathrm{KO}$ (Figure 1E and Supplementary Table S1). Although not associated with enriched pathways, THRSP was identified as a hub (Supplementary Table S7); three THRSP interacting partners, IGF1R, KRAS and PIK3CA, were mapped to enriched KEGG pathways "PI3K-Akt signaling pathway" and "FoxO signaling pathway"; two interacting partners, SMAD4 and TGFB1 were mapped to "Cell cycle" and "FoxO signaling pathway" and one interactor FGFR4 was mapped to "Cell cycle" and "PI3K-Akt signaling pathway" (Figure 5B).

HSP90AA1, a cell cycle and transcriptional regulatory chaperone, (identified as a bottleneck) was mapped to the enriched "PI3K-Akt signaling pathway" and was the only factor that interacted with both ARNTL and NPAS2 in the "Circadian rhythm” module (Figures 5A,B). Although Hsp90aa1 expression was unchanged in Cd151KO vs. WT, HSP90AA1 formed a vital link between the "Cell cycle| PI3-Akt signaling| aegingrelated FoxO signaling" module and the "Circadian rhythm" module (Figures 5A,B). 
A

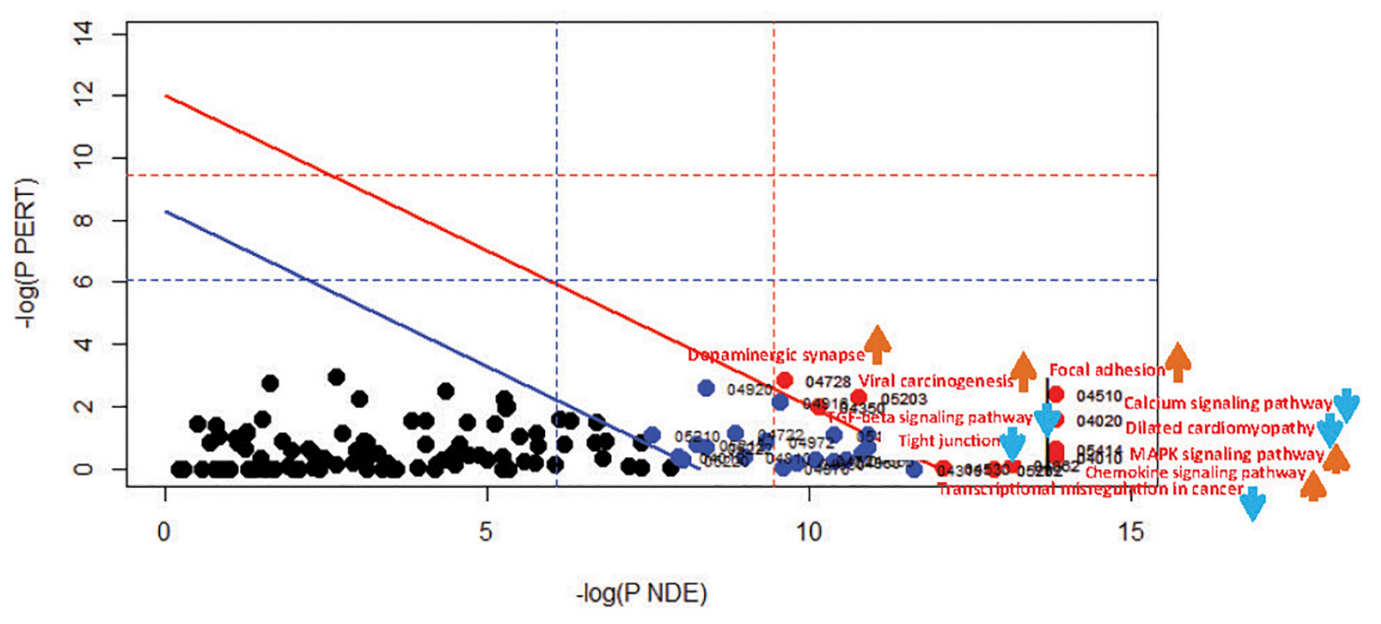

B

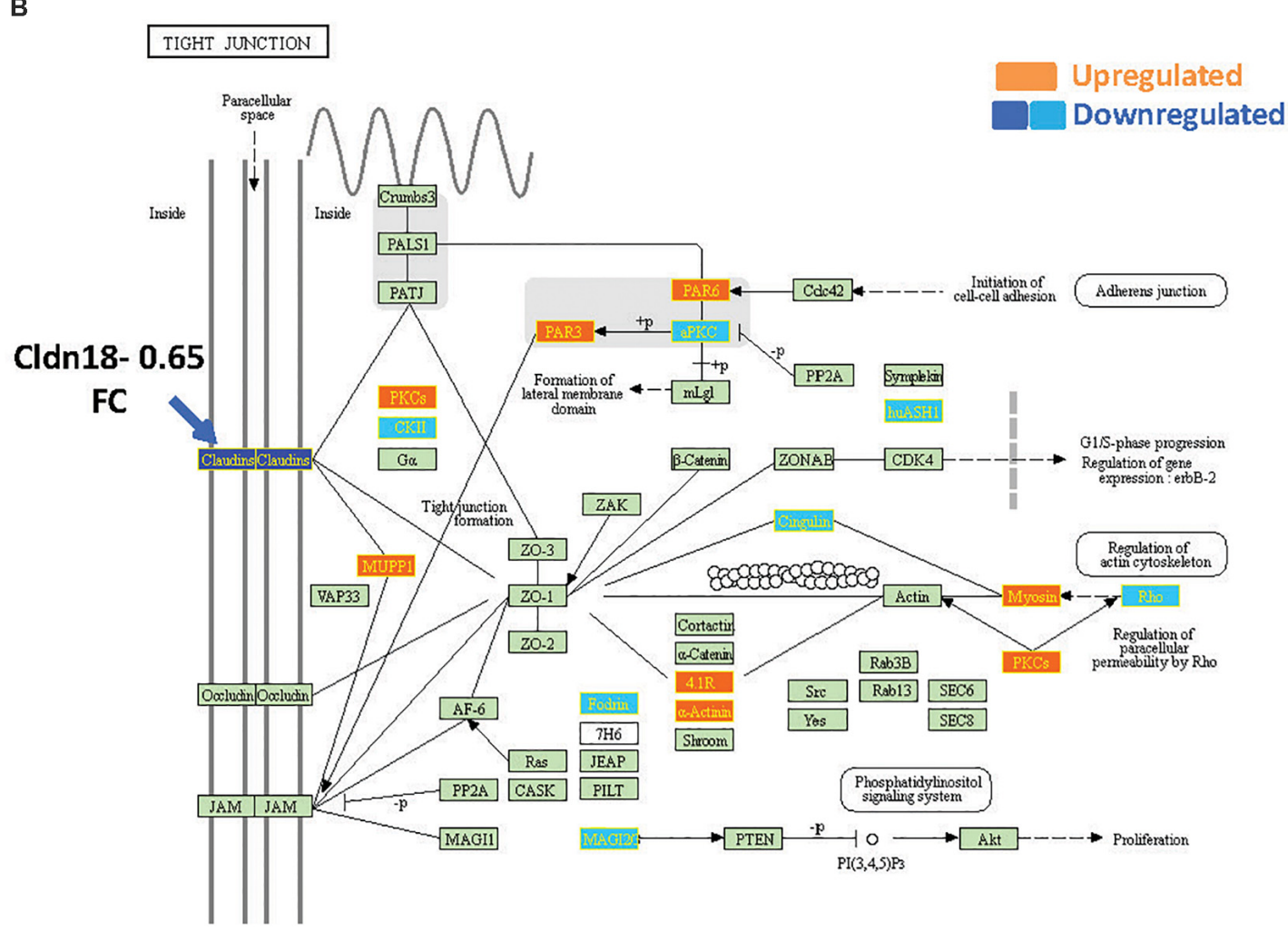

FIGURE 4 | SPIA identified pathway perturbations in Cd9KO. (A) SPIA two-way evidence plots for Cd9KO 1.2FC sDEGs. Significantly perturbed pathways are highlighted in red. "Activated" pathways are tagged with a red upward arrow and "Inhibited" pathways are tagged with a blue downward arrow. (B) KEGG pathway hsa04530: "Tight junction" was identified to be inhibited in Cd9KO. Upregulated genes are highlighted in orange and the downregulated genes are highlighted in dark blue (Claudins) and light blue (others), rectangles, respectively; their corresponding gene labels within the rectangles are highlighted in yellow.

\section{PPIN Analysis Highlighted a TNF-Signaling Mediated Pro-Apoptotic Signaling Related Module in Cd9KO}

Fas cell surface death receptor (Fas) (1.5FC) and Mapk10 (1.55FC) were elevated in Cd9KO (Supplementary Table S1). In Cd9KO-network, FAS, of the TNF-receptor superfamily that functions in extrinsic apoptosis pathway, was tagged as a bottleneck-hub (Supplementary Table S7), while MAPK10, a pro-apoptotic factor, known to be deleted in small-celllung-cancer (Kim et al., 2006), was identified as a bottleneck (Supplementary Table S7). FAS and MAPK10 were mapped to the enriched "TNF signaling pathway" ( $\left.p=2.8 \times 10^{-6}\right)$ (Supplementary Figure S5B and Supplementary Tables S8A,B) and emerged as key components of the inferred TNF signaling network (Figure 5C). 
A

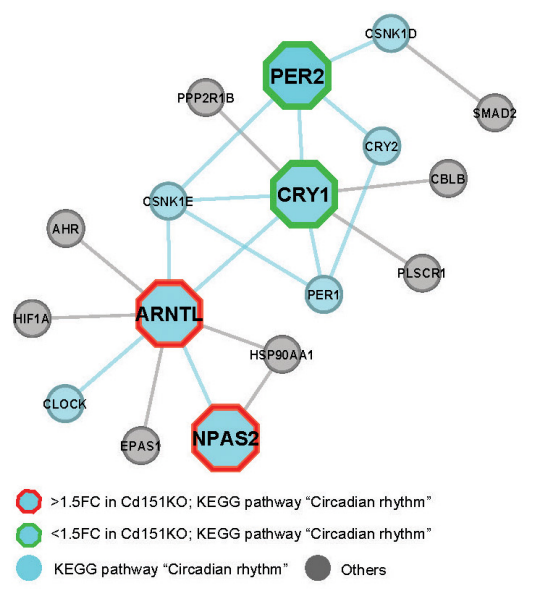

B

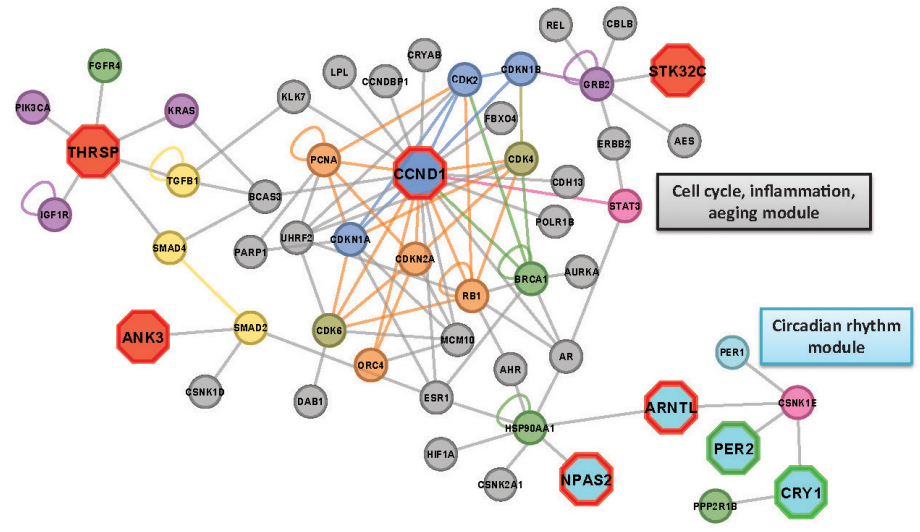

>1.5FC in CD151KO; "Cell cycle|PI3K-Akt signaling pathway|FoXo signaling pathway"

>1.5FC in CD151KO; "Circadian rhythm" <0.67FC in CD151KO; "Circadian rhythm"

$>1.5 \mathrm{FC}$ in CD151Ko "Cell cycle|PI3K-Akt signaling pathway|FoXo signaling pathway"

"Cell cycle|PI3K-Akt signaling pathway" "Cell cycle|FoxO signaling pathway"

"PI3K-Akt signaling pathway|FoXo signaling pathway" "Cell cycle"

"PI3K-Akt signaling pathway" "FoxO signaling pathway"

KEGG pathway "Circadian rhythm" Others

C

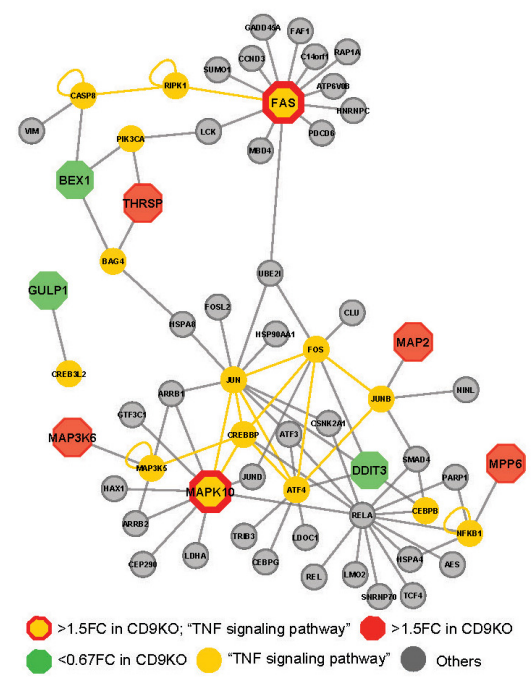

FIGURE 5 | (A) Cd151KO 1.5FC sDEGs orthologs and their interacting partners associated with Enriched KEGG pathway hsa04710: "Circadian rhythm." (B) Cd151KO 1.5FC sDEGs orthologs and their interacting partners associated with Enriched KEGG pathways hsa04110: "Cell cycle," hsa04710: "Circadian rhythm," hsa04068: "FoxO signaling pathway," and hsa04151: "PI3K-Akt signaling pathway." (C) Cd9KO 1.5FC sDEGs orthologs and their interacting partners associated with enriched KEGG pathway hsa04668: "TNF signaling pathway." 


\section{DISCUSSION}

By analyzing transcriptomic data from transgenic mice deficient in Cd151 (an in vivo model of IPF) or Cd9 (an in vivo model of emphysema), we obtained deeper insights into their functions in lung pathology. Our observations suggested that Cd151 and Cd9 modulate largely different signaling processes that have probably contributed to their contrasting roles in pulmonary pathophysiology. Specifically, Cd151-deletion resulted in elevated expression of genes involved in collagen deposition and ECM formation and dysregulation of genes associated with the circadian rhythm. Conversely, Cd9-deletion impacted genes associated with vascular remodeling and cell adhesion.

Circadian rhythm emerged as the premier process that was significantly impacted by Cd151-deletion. Circadian clock components Cry1, Per2, and Per3 were downregulated and the circadian regulators Arntl (Bmal1) and Npas2 were elevated in Cd151KO that manifested as the "Activation" of the "Circadian rhythm" pathway. Circadian clock dysfunctions are verifiably linked with a decline in lung function in chronic airway diseases, COPD and asthma (Chen et al., 2010; Pekovic-Vaughan et al., 2014; Sundar et al., 2015; Yao et al., 2015). Previously we had noted fibrotic changes in the lung, kidney and liver in Cd151KO, thereby implicating Cd151 in fibrosis in all the three organs (Tsujino et al., 2012). Our current analysis suggested that decreased Per2 expression following Cd151-deletion may be a prime determinant of the age-related pulmonary and multi-organ fibrosis in Cd151KO.

Cd151KO-network analysis highlighted HSP90AA1 as a probable "bridge" connecting the increased output of the circadian clock with the cell cycle, aeging and inflammationassociated factors. Indeed, HSP90AA1 was previously identified as an important crosslink between PPI-network modules representing cell cycle and signal transduction pathways in lung development (Yu et al., 2016). Thus, our analysis has illuminated a novel interplay between Cd151, circadian clock and cell cycle-related processes in maintaining lung homeostasis, the dysregulation of which may have significantly contributed to progressive multi-organ fibrotic phenotypes in Cd151KO. Circadian clock components, therefore, offer attractive targets for IPF therapy. Recently, the small-molecule modulators of circadian clock components have emerged as promising approaches to restore circadian rhythmicity in diseased conditions (Miller and Hirota, 2020). Specifically, a CLOCK-binding small molecule (CLK8) inhibited the CLOCKBMAL interaction and dimerization and the subsequent translocation of CLOCK into the nucleus, leading to the enhancement of the circadian rhythm (Doruk et al., 2020); CLK8 thus promises to be a useful means in therapeutic approaches seeking to correct dampened circadian rhythm in diseased conditions. Conversely, future studies that may enable the discovery of novel compounds that dampen the enhanced circadian activities, will have immense potential in the therapeutic interventions against pulmonary fibrosis.

Previously, Cd151-deletion resulted in ECM over-production in lung, in aberrant cell migration and a progressive fibrotic phenotype (Tsujino et al., 2012). Here, Cd151-deletion led to an upregulation of ECM- and collagen assembly-associated genes that are linked with disturbances in lung function. Our results, therefore, suggested that Cd151-deletion directly induced increased collagen deposition and ECM-remodeling that may eventually lead to progressive lung fibrosis.

Cd9-deletion is associated with diminished angiogenetic effects (Iwasaki et al., 2013); our observations suggested that these effects were likely to be mediated by dysregulation of circulatory system-associated genes Adipoq (Adiponectin), Fgg (fibrinogen gamma chain) Gclc (glutamate-cysteine ligase, catalytic subunit), Calca (Calcitonin) and Lox.

Among other examples, Glp1r (glucagon-like peptide 1 receptor), which is decreased in smokers with moderate COPD (Llinas et al., 2011), was decreased in Cd9KO. Conversely, Cd9deletion led to increased $M m p 13$ levels that are also elevated in COPD patients (Lee et al., 2009).

Furthermore, Cd9-deletion "Activated" "MAPK signaling pathway" and "Chemokine signaling pathway," both of which are consistent with increased lung inflammation and elevated proinflammatory cytokines in Cd9KO (Suzuki et al., 2009) and are also consistent with phenotypes in emphysema.

"TGF-beta signaling pathway" (mmu04350; pGFWER $=0.009108$ ) was "Inhibited" in Cd9KO. TGF- $\beta$ signaling is crucial in lung fibrosis and we had demonstrated that Cd151-deletion augmented TGF- $\beta$ signaling in the development of lung fibrosis (Tsujino et al., 2012). No studies have linked TGF$\beta$ signaling with CD9, in the context of emphysema. However, it was established that the cell surface protein EWI-2 negatively regulated TGF- $\beta$ signaling in melanoma by sequestering CD9 (and CD81) and thereby impeding complex formation between TGF $\beta$ receptor 1 (T $\beta R 1)$ and TGF $\beta$ receptor 2 (T $\beta R 2)$, leading to diminished TGF- $\beta$ signaling (Wang and Hemler, 2015). Our analysis suggested that the Cd9-deletion is likely to marginally enhance Decorin, which in turn may impede Tgfb binding with T $\beta R 1$ and T $\beta R 2$. Taken together with the observation that Eng, a glycoprotein involved with TGF- $\beta$ signaling, was also downregulated $(0.66 \mathrm{FC})$ in $\mathrm{Cd} 9 \mathrm{KO}$ (Supplementary Table S1), our results suggested for the first time that the loss of $\mathrm{Cd} 9$ activity may negatively impact TGF- $\beta$ signaling and the subsequent transitions in Cd9KO lungs. The observed decrease in TGF- $\beta$ signaling in $\mathrm{Cd} 9 \mathrm{KO}$ mice exhibiting an emphysema phenotype in our study is consistent with the previous studies that have discussed a decrease in TGF- $\beta$ signaling in human COPD (Wu et al., 2004; Chilosi et al., 2012).

Previously, the loss of Cd9 function exacerbated LPS-induced lung inflammation, chiefly via enhanced production of TNF- $\alpha$ and matrix metalloproteinases (Suzuki et al., 2009). Interestingly, in cigarette smoke-induced emphysema, impeding of Fasmediated apoptosis signaling attenuated smoking-induced lung injury in AECs (Kim et al., 2016). Cd9KO-network analysis suggested that Cd9-deletion may possibly trigger downstream TNF-signaling, chiefly via upregulation of Fas, leading to Fas receptor-induced death-inducing signaling complex (DISC) assembly, thereby significantly contributing to injury and cell death in AECs.

Despite considerable differences in $\mathrm{Cd} 151 \mathrm{KO}$ and $\mathrm{Cd} 9 \mathrm{KO}$ phenotypes, we observed overlaps between $\mathrm{Cd} 151 \mathrm{KO}$ and 
Cd9KO sDEGs. The overlapping sDEGs were broadly mapped to ECM (Adamts15, Eln, Lox), Vascular remodeling (Adipoq, Lox, and $M f_{s} d 2 a$ ), Lipid and hormone metabolism (Adipoq, Sptssb, Thrsp) and Transcription regulation (Tbx20, Tef, Thrsp) (Figure 1C and Supplementary Table S1). The overlaps were not limited to sDEGs alone; for instance "Focal Adhesion" (mmu04510) was "Activated" in Cd151KO and Cd9KO (Supplementary Table S3). Likewise, "Pathways in cancer," "MAPK signaling pathway," "PI3K-Akt signaling pathway" and "Adherens junction" were significantly enriched both in the Cd151KO- and Cd9KO-networks. An attractive explanation would be that these genes and pathways represent a "common core" of signaling cascades underlying both Cd151 and Cd9 functions in lung pathology and that this "common core" may also be potentially related with the elusive pathophysiology of CPFE.

\section{CONCLUSION}

Our analysis has provided novel insights into the contrasting roles of $\mathrm{Cd} 151$ and $\mathrm{Cd} 9$ in the pathogenesis of IPF and emphysema, respectively. We have also illuminated the genes and pathways that appeared to commonly underlie the functions of both Cd151 and Cd9 in lung pathology. Potentially the therapeutic targeting of dysregulated Cd151- and Cd9-signaling may contribute to improved and more effective therapy for pulmonary diseases.

\section{DATA AVAILABILITY STATEMENT}

The microarray data associated with this study has been deposited in GEO (accession number: GSE 159236).

\section{ETHICS STATEMENT}

The animal study was reviewed and approved by Animal Care and Use Committee of Osaka University.

\section{REFERENCES}

Albrecht, U. (2012). Timing to perfection: the biology of central and peripheral circadian clocks. Neuron 74, 246-260. doi: 10.1016/j.neuron.2012.04.006

Benjamini, Y., Drai, D., Elmer, G., Kafkafi, N., and Golani, I. (2001). Controlling the false discovery rate in behavior genetics research. Behav. Brain Res 125, 279-284. doi: 10.1016/s0166-4328(01)00297-2

Brightling, C. E. (2013). Biomarkers that predict and guide therapy for exacerbations of chronic obstructive pulmonary disease. Ann. Am. Thoracic Soc. 10(Suppl.), S214-S219.

Charrin, S., Jouannet, S., Boucheix, C., and Rubinstein, E. (2014). Tetraspanins at a glance. J. Cell Sci. 127, 3641-3648. doi: 10.1242/jcs. 154906

Chen, P., Han, Z., Yang, P., Zhu, L., Hua, Z., and Zhang, J. (2010). Loss of clock gene mPer2 promotes liver fibrosis induced by carbon tetrachloride. Hepatol. Res. 40, 1117-1127. doi: 10.1111/j.1872-034x.2010.00695.x

Chen, Y. A., Tripathi, L. P., and Mizuguchi, K. (2011). TargetMine, an integrated data warehouse for candidate gene prioritisation and target discovery. PLoS One 6:e17844. doi: 10.1371/journal.pone.0017844.g004

\section{AUTHOR CONTRIBUTIONS}

LT, MI, YT, and KM designed the study, were responsible for overall data analysis and interpretation, and wrote the manuscript. LT, MI, YT, KT, YK, AK, and KM were responsible for data gathering and validation. LT and MI performed the computational data analysis. All authors read and approved the final version of the manuscript.

\section{FUNDING}

This work was in part supported by Grants-in-Aid for Scientific Research from the Japan Agency for Medical Research and Development (Grant Number 19ak0101068h0003; “The adjuvant database project” Grant Number 16ak0101010h0005) and from the Japan Society for the Promotion of Science (Grant Number 17K07268) to KM, Grant-in-Aid for Scientific Research from the Ministry of Health, Labor, and Welfare (Grant Number 19AC5001) to KM, a Grant-in-Aid for Scientific Research from the Ministry of Health, Labor, and Welfare (No. JP19K08650) to YT, and a Grant from Uehara Memorial Foundation to YT.

\section{ACKNOWLEDGMENTS}

The authors thank the members of the Mizuguchi Laboratory for their critical review of the study and manuscript.

\section{SUPPLEMENTARY MATERIAL}

The Supplementary Material for this article can be found online at: https://www.frontiersin.org/articles/10.3389/fgene. 2020.585998/full\#supplementary-material

Chen, Y. A., Tripathi, L. P., and Mizuguchi, K. (2016). An integrative data analysis platform for gene set analysis and knowledge discovery in a data warehouse framework. Database 2016:baw009. doi: 10.1093/database/ba w009

Chilosi, M., Poletti, V., and Rossi, A. (2012). The pathogenesis of COPD and IPF: distinct horns of the same devil? Respirat. Res. 13:3. doi: 10.1186/1465-992113-3

Cline, M. S., Smoot, M., Cerami, E., Kuchinsky, A., Landys, N., Workman, C., et al. (2007). Integration of biological networks and gene expression data using Cytoscape. Nat. Prot. 2, 2366-2382.

Cottin, V., Nunes, H., Brillet, P. Y., Delaval, P., Devouassoux, G., Tillie-Leblond, I., et al. (2005). Combined pulmonary fibrosis and emphysema: a distinct underrecognised entity. Eur. Respirat. J. 26, 586-593. doi: 10.1183/09031936. 05.00021005

Doruk, Y. U., Yarparvar, D., Akyel, Y. K., Gul, S., Taskin, A. C., Yilmaz, F., et al. (2020). A CLOCK-binding small molecule disrupts the interaction between CLOCK and BMAL1 and enhances circadian rhythm amplitude. J. Biol. Chem. 295, 3518-3531. doi: 10.1074/jbc.ra119.011332 
Fabregat, A., Sidiropoulos, K., Garapati, P., Gillespie, M., Hausmann, K., Haw, R., et al. (2016). The Reactome pathway Knowledgebase. Nucl. Acids Res. 44, D481-D487.

Garavoglia, M., Borghi, F., Bianco, S., Mauthe Degerfeld, M., Gentilli, S., Gattolin, A., et al. (1993). Ileo-colon-rectoplasty. An experimental morpho-functional study on the pig. Panm. Med. 35, 28-32.

Gene Ontology Consortium, (2015). Gene ontology consortium: going forward. Nucl. Acids Res. 43, D1049-D1056.

Haque, R., Ali, F. G., Biscoglia, R., Abey, J., Weller, J., Klein, D., et al. (2010). CLOCK and NPAS2 have overlapping roles in the circadian oscillation of arylalkylamine $\mathrm{N}$-acetyltransferase mRNA in chicken cone photoreceptors. J. Neurochem. 113, 1296-1306.

Iwasaki, T., Takeda, Y., Maruyama, K., Yokosaki, Y., Tsujino, K., Tetsumoto, S., et al. (2013). Deletion of tetraspanin CD9 diminishes lymphangiogenesis in vivo and in vitro. J. Biol. Chem. 288, 2118-2131. doi: 10.1074/jbc.m112.424291

Jarvinen, T. A., and Prince, S. (2015). Decorin: a growth factor antagonist for tumor growth inhibition. Biomed Res. Int. 2015, 654765.

Jin, Y., Takeda, Y., Kondo, Y., Tripathi, L. P., Kang, S., Takeshita, H., et al. (2018). Double deletion of tetraspanins CD9 and CD81 in mice leads to a syndrome resembling accelerated aging. Sci. Rep. 8:5145.

Kanehisa, M., Sato, Y., Kawashima, M., Furumichi, M., and Tanabe, M. (2016). KEGG as a reference resource for gene and protein annotation. Nucl. Acids Res. 44, D457-D462.

Kim, C., Lee, J. M., Park, S. W., Kim, K. S., Lee, M. W., Paik, S., et al. (2016). Attenuation of cigarette smoke-induced emphysema in mice by apolipoprotein A-1 overexpression. Am. J. Respirat. Cell Mol. Biol. 54, 91-102. doi: 10.1165/ rcmb.2014-0305oc

Kim, Y. H., Girard, L., Giacomini, C. P., Wang, P., Hernandez-Boussard, T., and Tibshirani, R. E. (2006). Combined microarray analysis of small cell lung cancer reveals altered apoptotic balance and distinct expression signatures of MYC family gene amplification. Oncogene 25, 130-138. doi: 10.1038/sj.onc.120 8997

Kumari, S., Devi, G., Badana, A., Dasari, V. R., and Malla, R. R. (2015). CD151-A Striking Marker for Cancer Therapy. Biomark. Cancer 7, 7-11.

Landgraf, D., Wang, L. L., Diemer, T., and Welsh, D. K. (2016). NPAS2 Compensates for Loss of CLOCK in Peripheral Circadian Oscillators. PLoS Genet. 12:e1005882. doi: 10.1371/journal.pgen.1005882

Langfelder, P., and Horvath, S. (2008). WGCNA: an R package for weighted correlation network analysis. BMC Bioinform. 9:559. doi: 10.1186/1471-21059-559

Lee, E. J., In, K. H., Kim, J. H., Lee, S. Y., Shin, C., Shim, J. J., et al. (2009). Proteomic analysis in lung tissue of smokers and COPD patients. Chest 135, 344-352. doi: 10.1378/chest.08-1583

Llinas, L., Peinado, V. I., Ramon Goni, J., Rabinovich, R., Pizarro, S., RodriguezRoisin, R., et al. (2011). Similar gene expression profiles in smokers and patients with moderate COPD. Pulm. Pharmacol. Ther. 24, 32-41. doi: 10.1016/j.pupt. 2010.10.010

Miller, M., Cho, J. Y., Pham, A., Ramsdell, J., and Broide, D. H. (2009). Adiponectin and functional adiponectin receptor 1 are expressed by airway epithelial cells in chronic obstructive pulmonary disease. J. Immunol. 182, 684-691. doi: 10.4049/jimmunol.182.1.684

Miller, S., and Hirota, T. (2020). Pharmacological Interventions to Circadian Clocks and Their Molecular Bases. J. Mol. Biol. 432, 3498-3514. doi: 10.1016/j. jmb.2020.01.003

Peek, C. B., Ramsey, K. M., Levine, D. C., Marcheva, B., Perelis, M., and Bass, J. (2015). Circadian regulation of cellular physiology. Methods Enzymol. 552, 165-184. doi: 10.1016/bs.mie.2014.10.006

Pekovic-Vaughan, V., Gibbs, J., Yoshitane, H., Yang, N., Pathiranage, D., Guo, B., et al. (2014). The circadian clock regulates rhythmic activation of the NRF2/glutathione-mediated antioxidant defense pathway to modulate pulmonary fibrosis. Genes Dev. 28, 548-560. doi: 10.1101/gad.2370 81.113

Raghu, G., Collard, H. R., Egan, J. J., Martinez, F. J., Behr, J., Brown, K. K., et al. (2011). An official ATS/ERS/JRS/ALAT statement: idiopathic pulmonary fibrosis: evidence-based guidelines for diagnosis and management. Am. J. Respirat. Crit. Care Med. 183, 788-824. doi: 10.1164/rccm.2009-040gl

Schlingmann, B., Molina, S. A., and Koval, M. (2015). Claudins: Gatekeepers of lung epithelial function. Semin. Cell Dev. Biol. 42, 47-57. doi: 10.1016/j.semcdb. 2015.04.009

Shimobaba, S., Taga, S., Akizuki, R., Hichino, A., Endo, S., Matsunaga, T., et al. (2016). Claudin-18 inhibits cell proliferation and motility mediated by inhibition of phosphorylation of PDK1 and Akt in human lung adenocarcinoma A549 cells. Biochim. Biophys. Acta 1863, 1170-1178. doi: 10.1016/j.bbamcr.2016.02.015

Sundar, I. K., Yao, H., Sellix, M. T., and Rahman, I. (2015). Circadian clockcoupled lung cellular and molecular functions in chronic airway diseases. Am. J. Respirat. Cell Mol. biology 53, 285-290. doi: 10.1165/rcmb.2014-0476tr

Suzuki, M., Tachibana, I., Takeda, Y., He, P., Minami, S., Iwasaki, T., et al. (2009). Tetraspanin CD9 negatively regulates lipopolysaccharide-induced macrophage activation and lung inflammation. J. Immunol. 182, 6485-6493. doi: 10.4049/ jimmunol.0802797

Takeda, Y., He, P., Tachibana, I., Zhou, B., Miyado, K., Kaneko, H., et al. (2008). Double deficiency of tetraspanins CD9 and CD81 alters cell motility and protease production of macrophages and causes chronic obstructive pulmonary disease-like phenotype in mice. J. Biol. Chem. 283, 26089-26097. doi: 10.1074/ jbc.m801902200

Tarca, A. L., Draghici, S., Khatri, P., Hassan, S. S., Mittal, P., Kim, J. S., et al. (2009). A novel signaling pathway impact analysis. Bioinform. 25, 75-82. doi: 10.1093/bioinformatics/btn577

Termini, C. M., and Gillette, J. M. (2017). Tetraspanins function as regulators of cellular signaling. Front. Cell Dev. Biol. 5:34. doi: 10.3389/fcell.2017.00034

Tsujino, K., Takeda, Y., Arai, T., Shintani, Y., Inagaki, R., and Saiga, H. (2012). Tetraspanin CD151 protects against pulmonary fibrosis by maintaining epithelial integrity. Am. J. Respirat. Crit. Care Med. 186, 170-180. doi: 10.1164/ rccm.201201-0117oc

Vogelmeier, C. F., Criner, G. J., Martinez, F. J., Anzueto, A., Barnes, P. J., Bourbeau, J., et al. (2017). Global Strategy for the Diagnosis, Management, and Prevention of Chronic Obstructive Lung Disease 2017 Report. GOLD Executive Summary. . Am. J. Respirat. Crit. Care Med. 195, 557-582.

Wang, H. X., and Hemler, M. E. (2015). Novel impact of EWI-2, CD9, and CD81 on TGF-beta signaling in melanoma. Mol. Cell. Oncol. 2:e1030536. doi: 10.1080/ 23723556.2015.1030536

Wu, L., Chau, J., Young, R. P., Pokorny, V., Mills, G. D., Hopkins, R., et al. (2004). Transforming growth factor-betal genotype and susceptibility to chronic obstructive pulmonary disease. Thorax 59, 126-129. doi: 10.1136/thorax.2003. 005769

Yao, H., Sundar, I. K., Huang, Y., Gerloff, J., Sellix, M. T., Sime, P. J., et al. (2015). Disruption of Sirtuin 1-mediated control of circadian molecular clock and inflammation in chronic obstructive pulmonary disease. Am. J. Respir. Cell Mol. Biol. 53, 782-792. doi: 10.1165/rcmb.2014-0474oc

Yu, X., Feng, L., Han, Z., Wu, B., Wang, S., Xiao, Y., et al. (2016). Crosstalk of dynamic functional modules in lung development of rhesus macaques. Mol. Biosyst. 12, 1342-1349. doi: 10.1039/c5mb00881f

Conflict of Interest: YK was employed by the company Sumitomo Dainippon Pharma Co., Ltd.

The remaining authors declare that the research was conducted in the absence of any commercial or financial relationships that could be construed as a potential conflict of interest.

Copyright (c) 2020 Tripathi, Itoh, Takeda, Tsujino, Kondo, Kumanogoh and Mizuguchi. This is an open-access article distributed under the terms of the Creative Commons Attribution License (CC BY). The use, distribution or reproduction in other forums is permitted, provided the original author(s) and the copyright owner(s) are credited and that the original publication in this journal is cited, in accordance with accepted academic practice. No use, distribution or reproduction is permitted which does not comply with these terms. 